\title{
Carbaporphyrinoid chemistry has a silver lining! Silver(III) oxybenzi-, oxynaphthi-, tropi- and benzocarbaporphyrins
}

Timothy D. Lash, ${ }^{*}$ Jane M. Rasmussen, Katrina M. Bergman and Denise A. Colby

Department of Chemistry, Illinois State University, Normal, Illinois 61790-4160

E-mail: tdlash@ilstu.edu

Supplementary Materials (5 pages)

\section{Sample Procedures:}

NMR spectra were obtained on a Varian Gemini $400 \mathrm{MHz}$ NMR spectrometer and recorded in ppm relative to $\mathrm{CDCl}_{3}$ (residual chloroform at $7.26 \mathrm{ppm}$ in proton $\mathrm{NMR}$ and $\mathrm{CDCl}_{3}$ triplet at $77.23 \mathrm{ppm}$ in carbon-13 NMR spectra). UV-vis spectra were recorded on a Varian Cary UV spectrophotometer. EI mass spectral determinations were made at the Mass Spectral Laboratory, School of Chemical Sciences, University of Illinois at Urbana-Champaign, supported in part by a grant from the National Institute of General Medical Sciences (GM 27029). Elemental analyses were obtained from the School of Chemical Sciences Microanalysis Laboratory at the University of Illinois. 
9,13,14,18-Tetraethyl-8,19-dimethyl-2-oxynaphthiporphyrin (16): Tripyrrane dicarboxylic acid 15 (100.2 mg) was stirred with TFA (1 mL) under an atmosphere of nitrogen for 5 min. The solution was diluted with dichloromethane $(19 \mathrm{~mL})$, followed immediately by the addition of 4-methoxynaphthalene-1,3-dicarbaldehyde $(\mathbf{1 4} ; 44.5 \mathrm{mg})$, and the mixture was stirred under nitrogen, in the dark, for a further $3 \mathrm{~h}$. After neutralization by the dropwise addition of triethylamine, DDQ $(51 \mathrm{mg})$ was added and the resulting solution was stirred in the dark for an additional $1 \mathrm{~h}$. The mixture was washed with water and chromatographed on Grade 3 alumina, eluting first with dichloromethane and then with chloroform. A deep green fraction was collected with chloroform and recrystallized from chloroform-methanol to give the porphyrin analogue (39.4 mg; 35\%) as sparkling purple needles, mp 283-284 ${ }^{\circ} \mathrm{C}$; IR (KBr): v 3343 (w, NH str.), $1630 \mathrm{~cm}^{-1}$ (s, C=O str.); UV-Vis $\left(\mathrm{CHCl}_{3}\right): \lambda_{\max }\left(\log _{10} \varepsilon\right) 431$ (5.29), 450 (4.96), 539 (3.94), 581 (4.51), 622 (3.97), 687 (3.36); UV-Vis (1\% TFA-CHCl $): \lambda_{\max }\left(\log _{10} \varepsilon\right) 317$ (4.62), 437 (5.31), 464 (4.94) 561 (3.99), 611 (4.37), 681 (3.60); ${ }^{1} \mathrm{H}$ NMR $\left(\mathrm{CDCl}_{3}\right): \delta$-7.42 (1H, s), $1.69(3 \mathrm{H}, \mathrm{t}, J=7.6 \mathrm{~Hz}), 1.74-1.80$ (9H, 3 overlapping triplets), 3.40 (3H, s), 3.57 (3H, s), 3.743.81 (4H, 2 overlapping quartets), $3.84(2 \mathrm{H}, \mathrm{q}, J=7.6 \mathrm{~Hz}), 3.93(2 \mathrm{H}, \mathrm{q}, J=7.7 \mathrm{~Hz}), 7.73(1 \mathrm{H}$, $\mathrm{t}, J=7.2 \mathrm{~Hz}), 7.96\left(1 \mathrm{H}, \mathrm{dt},{ }^{4} J=1.2 \mathrm{~Hz},{ }^{3} J=7 \mathrm{~Hz}\right), 8.92\left(1 \mathrm{H}, \mathrm{dd},{ }^{3} J=7 \mathrm{~Hz},{ }^{4} J=1.2 \mathrm{~Hz}\right), 8.96$ $(1 \mathrm{H}, \mathrm{d}, J=8 \mathrm{~Hz}), 9.27(1 \mathrm{H}, \mathrm{s}), 9.33(1 \mathrm{H}, \mathrm{s}), 9.94(1 \mathrm{H}, \mathrm{s}), 10.65(1 \mathrm{H}, \mathrm{s}) ;{ }^{13} \mathrm{C} \mathrm{NMR}\left(\mathrm{CDCl}_{3}\right): \delta$ $11.8,11.9,17.2,18.4,19.5,19.6,19.9,94.2,94.6,103.0,113.6,120.1,123.0,124.9,127.7$, $128.8,132.1,132.7,133.4,134.0,135.3,136.6,137.1,137.8,138.7,140.6,144.6,144.7$, 154.3, 154.8, 187.3; HR MS (EI): calcd for $\mathrm{C}_{36} \mathrm{H}_{37} \mathrm{~N}_{3} \mathrm{O}: \mathrm{m} / \mathrm{z}$ 527.2937; found: 527.2932.

\section{[9,13,14,18-Tetraethyl-8,19-dimethyl-2-oxynaphthiporphyrinato]silver(III) (17).}

A suspension of silver(I) acetate $(40.5 \mathrm{mg})$ in methanol $(10 \mathrm{~mL})$ was added to a solution of 9,13,14,18-tetraethyl-8,19-dimethyl-2-oxynaphthiporphyrin $(20.1 \mathrm{mg})$ in chloroform (20 mL) and the resulting mixture stirred at room temperature under nitrogen for $16 \mathrm{~h}$. The mixture was washed with water (x2), dried over sodium sulfate, and evaporated under reduced pressure. The greenish-purple residue was purified by column chromatography on grade 3 alumina, 
eluting with dichloromethane and then chloroform. Evaporation of the green fractions and recrystallization from chloroform-hexanes gave the silver chelate $(21.1 \mathrm{mg}, 87 \%)$ as dark greyish green crystals, mp 274-275 ${ }^{\circ} \mathrm{C}$; IR (KBr): v 1642 (s), $1619 \mathrm{~cm}^{-1}$ (s, C=O str.); UV-Vis $\left(\mathrm{CHCl}_{3}\right): \lambda_{\max }\left(\log _{10} \varepsilon\right) 456$ (4.975), $522(3.70), 593$ (4.39), $616 \mathrm{~nm}(4.075) ;{ }^{1} \mathrm{H} \mathrm{NMR}\left(\mathrm{CDCl}_{3}\right): \delta$ $1.54(3 \mathrm{H}, \mathrm{t}, J=7.8 \mathrm{~Hz}), 1.65-1.72(9 \mathrm{H}, 3$ overlapping triplets), $2.98(3 \mathrm{H}, \mathrm{s}), 3.33(3 \mathrm{H}, \mathrm{s}), 3.47$ $(2 \mathrm{H}, \mathrm{q}, J=7.8 \mathrm{~Hz}), 3.60-3.71(6 \mathrm{H}, 3$ overlapping quartets), $7.71(1 \mathrm{H}, \mathrm{t}, J=7.2 \mathrm{~Hz}), 7.85(1 \mathrm{H}$, dt, $J=1.4,7.4 \mathrm{~Hz}), 8.27(1 \mathrm{H}, \mathrm{d}, J=8 \mathrm{~Hz}), 8.73(1 \mathrm{H}, \mathrm{dd}, J=1.4,7.8 \mathrm{~Hz}), 8.89(1 \mathrm{H}, \mathrm{s}), 9.06$ $(1 \mathrm{H}, \mathrm{s}), 9.14(1 \mathrm{H}, \mathrm{s}), 9.90(1 \mathrm{H}, \mathrm{s}) ;{ }^{13} \mathrm{C} \mathrm{NMR}\left(\mathrm{CDCl}_{3}\right): \delta 11.4,11.8,17.2,17.3,18.2,19.7(2)$, 20.1, 20.3, 95.8, 95.9, 107.5, 109.9, 110.8, 113.6, 126.9, 127.7, 128.4, 131.6, 132.4, 133.0, 133.5, 135.1, 136.9, 138.1, 138.5, 139.1, 141.1, 141.3, 141.4, 142.5, 143.0, 191.6; FD MS: m/z (rel. int.) 635.2 (7.3), 634.2 (35), $633.2(90), 632.1(40), 631.2\left(100, \mathrm{M}^{+}\right)$.

\section{[9,13,14,18-Tetraethyl-8,19-dimethyl-2-oxybenziporphyrinato]silver(III) (13a).}

A suspension of silver(I) acetate $(40 \mathrm{mg})$ in methanol $(10 \mathrm{~mL})$ was added to a solution of 9,13,14,18-tetraethyl-8,19-dimethyl-2-oxybenziporphyrin (20.0 mg) in chloroform (20 mL) and the resulting mixture stirred at room temperature for $16 \mathrm{~h}$. The mixture was washed with water and evaporated under reduced pressure. The residue was purified by column chromatography on Grade 3 alumina, eluting with chloroform and then 1\% methanol-chloroform. Evaporation of the green fractions afforded a dark residue. Recrystallization from chloroform-methanol gave the silver chelate $(23.5 \mathrm{mg}, 96 \%)$ as a dark purple powder, $\mathrm{mp}>300{ }^{\circ} \mathrm{C}$; IR $(\mathrm{KBr}): v 1641$ (s), $1619 \mathrm{~cm}^{-1}$ (s, $\mathrm{C}=\mathrm{O}$ str.); UV-Vis $\left(\mathrm{CHCl}_{3}\right): \lambda_{\max }\left(\log _{10} \varepsilon\right) 336$ (4.51), 456 (4.94), 524 (3.55), 608 (4.40), $629 \mathrm{~nm}(4.20) ;{ }^{1} \mathrm{H} \mathrm{NMR}\left(\mathrm{CDCl}_{3}\right): \delta 1.60(3 \mathrm{H}, \mathrm{t}, J=8 \mathrm{~Hz}), 1.64-1.73(9 \mathrm{H}, 3$ overlapping triplets), $3.10(3 \mathrm{H}, \mathrm{s}), 3.31(3 \mathrm{H}, \mathrm{s}), 3.62(2 \mathrm{H}, \mathrm{q}, J=8 \mathrm{~Hz}), 3.67-3.75(6 \mathrm{H}, 3$ overlapping quartets), $7.32(1 \mathrm{H}, \mathrm{d}, J=8.4 \mathrm{~Hz}), 8.44(1 \mathrm{H}, \mathrm{d}, J=8.4 \mathrm{~Hz}), 8.69(1 \mathrm{H}, \mathrm{s}), 9.01(1 \mathrm{H}$, s), $9.13(1 \mathrm{H}, \mathrm{s}), 10.23(1 \mathrm{H}, \mathrm{s})$; HRMS (EI): calcd for $\mathrm{C}_{32} \mathrm{H}_{32} \mathrm{~N}_{3} \mathrm{OAg}$ : 581.1610; found: 581.1608. Anal. Calcd for $\mathrm{C}_{32} \mathrm{H}_{32} \mathrm{~N}_{3} \mathrm{OAg} .{ }_{8}{ }_{8} \mathrm{CHCl}_{3}$ : C, 64.51; H, 5.37; N, 7.02. Found: C, 64.56; H, 5.38; N, 6.95 . 
[9,18-Diethyl-8,19-dimethyl-13,14-diphenyl-2-oxybenziporphyrinato]silver(III) (13b). A suspension of silver(I) acetate $(40 \mathrm{mg})$ in methanol $(10 \mathrm{~mL})$ was added to a solution of 9,18 diethyl-8,19-dimethyl-13,14-diphenyl-2-oxybenziporphyrin $(20.0 \mathrm{mg})$ in chloroform $(20 \mathrm{~mL})$ and the resulting mixture stirred at room temperature for $16 \mathrm{~h}$. The mixture was washed with water and evaporated under reduced pressure. The residue was purified by column chromatography on Grade 3 alumina, eluting with chloroform and then $1 \%$ methanolchloroform. Evaporation of the green fractions afforded a dark residue. Recrystallization from chloroform-methanol gave the silver chelate $(22.5 \mathrm{mg}, 95 \%)$ as a dark green powder, $\mathrm{mp}>300$ ${ }^{\circ} \mathrm{C}$; IR (KBr): v 1639 (s), $1618 \mathrm{~cm}^{-1}$ (s, C=O str.); UV-Vis $\left(\mathrm{CHCl}_{3}\right): \lambda_{\max }\left(\log _{10} \varepsilon\right) 344$ (4.59), 458 (4.96), 526 (3.57), 614 (4.48), $638 \mathrm{~nm} \mathrm{(4.31);}{ }^{1} \mathrm{H}$ NMR $\left(\mathrm{CDCl}_{3}\right): \delta$ 1.67-1.74 (6H, 2 overlapping triplets), $3.30(3 \mathrm{H}, \mathrm{s}), 3.43(3 \mathrm{H}, \mathrm{s}), 3.72-3.79(4 \mathrm{H}, 2$ overlapping quartets), 7.31 $(1 \mathrm{H}, \mathrm{d}, J=8 \mathrm{~Hz}), 7.65(2 \mathrm{H}, \mathrm{t}, J=7.6 \mathrm{~Hz}), 7.73(4 \mathrm{H}, \mathrm{t}, J=7.4 \mathrm{~Hz}), 7.99-8.02(4 \mathrm{H}, \mathrm{m}), 8.47$ $(1 \mathrm{H}, \mathrm{d}, J=8 \mathrm{~Hz}), 8.87(1 \mathrm{H}, \mathrm{s}), 9.69(1 \mathrm{H}, \mathrm{s}), 9.71(1 \mathrm{H}, \mathrm{s}), 10.32(1 \mathrm{H}, \mathrm{s}) ;{ }^{1} \mathrm{H}$ NMR $\left(\mathrm{d}_{4}-\mathrm{MeOH}-\right.$ $\left.\mathrm{CDCl}_{3}, 50{ }^{\circ} \mathrm{C}\right): \delta 1.56(3 \mathrm{H}, \mathrm{t}, J=7.6 \mathrm{~Hz}), 1.63(3 \mathrm{H}, \mathrm{t}, J=7.6 \mathrm{~Hz}), 3.02(3 \mathrm{H}, \mathrm{s}), 3.24(3 \mathrm{H}, \mathrm{s})$, $3.55(2 \mathrm{H}, \mathrm{q}, J=7.6 \mathrm{~Hz}), 3.62(2 \mathrm{H}, \mathrm{q}, J=7.6 \mathrm{~Hz}), 7.04(1 \mathrm{H}, \mathrm{d}, J=8.4 \mathrm{~Hz}), 7.60(2 \mathrm{H}, \mathrm{t}, J=7.6$ Hz), 7.66-7.71 (4H, m), 7.92-7.96 (4H, m), $8.02(1 \mathrm{H}, \mathrm{d}, J=8.8 \mathrm{~Hz}), 8.28(1 \mathrm{H}, \mathrm{s}), 9.43(1 \mathrm{H}, \mathrm{s})$, $9.46(1 \mathrm{H}, \mathrm{s}), 9.92(1 \mathrm{H}, \mathrm{s}) ;{ }^{13} \mathrm{C} \mathrm{NMR}\left(\mathrm{d}_{4}-\mathrm{MeOH}-\mathrm{CDCl}_{3}, 50{ }^{\circ} \mathrm{C}\right): \delta \quad 11.2,11.6,16.7,16.8,20.1$, $20.3,29.8,99.9,101.2,113.5,115.9,113.5,115.9,125.7,127.9,128.8,132.5,135.3,139.1$ (due to very the very low solubility of the silver complex not all of the carbon-13 resonances could be identified); HRMS (EI): calcd for $\mathrm{C}_{40} \mathrm{H}_{32} \mathrm{~N}_{3} \mathrm{OAg}$ : 677.1596; found: 677.1592. Anal. Calcd for $\mathrm{C}_{40} \mathrm{H}_{32} \mathrm{~N}_{3} \mathrm{OAg}$ : C, 70.80; H, 4.75; N, 6.19. Found: C, 70.83; H, 4.65; N, 6.22.

[10,14,15,19-Tetraethyl-9,20-dimethyltropiporphyrinato]silver(III) (11a). Silver(I) acetate (11.0 mg) was added to a solution of 10,14,15,19-tetraethyl-9,20-dimethyltropiporphyrin (8.8 $\mathrm{mg})$ and DBU (3 drops) in pyridine $(15 \mathrm{~mL})$ and the mixture stirred overnight in the dark at room temperature. The mixture was diluted with chloroform, washed with water, and the 
solvent removed under reduced pressure. The residue was chromatographed on a silica flash column eluting with dichloromethane. A green fraction was collected and recrystallized from chloroform-hexanes to give the silver(III) complex $(5.1 \mathrm{mg}, 47 \%)$ as dark crystals, $\mathrm{mp}>300{ }^{\circ} \mathrm{C}$; $\mathrm{UV}-\mathrm{Vis}\left(\mathrm{CHCl}_{3}\right): \lambda_{\max }\left(\log _{10} \varepsilon\right) 381$ (4.53), 432 (4.50), 499 (3.91), 533 (3.69), $604 \mathrm{~nm}(3.92) ;{ }^{1} \mathrm{H}$ NMR $\left(\mathrm{CDCl}_{3}\right): \delta 1.67(6 \mathrm{H}, \mathrm{t}, J=7.6 \mathrm{~Hz}), 1.76(6 \mathrm{H}, \mathrm{t}, J=7.6 \mathrm{~Hz}), 3.14(6 \mathrm{H}, \mathrm{s}), 3.72-3.76(8 \mathrm{H}$, m), 6.89-6.92 (2H, m), 7.14-7.17 (2H, m), $7.99(2 \mathrm{H}, \mathrm{s}), 9.12(2 \mathrm{H}, \mathrm{s}) ;{ }^{13} \mathrm{C} \mathrm{NMR}\left(\mathrm{CDCl}_{3}\right): \delta 11.5$, $17.1,18.1,19.7,20.1,95.4,100.8,120.7,128.5,135.4$ (2), 136.9, 142.6, 143.0, 143.1, 144.4 (2); HRMS (EI): calcd for $\mathrm{C}_{33} \mathrm{H}_{34} \mathrm{~N}_{3} \mathrm{Ag}$ : 579.1801; found: 579.1804. Anal. Calcd for $\mathrm{C}_{33} \mathrm{H}_{34} \mathrm{~N}_{3} \mathrm{Ag}: \mathrm{C}, 68.28 ; \mathrm{H}, 5.90 ; \mathrm{N}, 7.24$. Found: C, 68.20; H, 5.86; N, 7.09.

[10,19-Diethyl-9,20-dimethyl-14,15-diphenyltropiporphyrinato]silver(III) (11b). Silver(I) acetate (12.4 mg) was added to a solution of 10,19-tetraethyl-9,20-dimethyl-14,15diphenyltropiporphyrin $(8.8 \mathrm{mg})$ and DBU (3 drops) in pyridine $(15 \mathrm{~mL})$ and the mixture stirred overnight in the dark at room temperature. The mixture was diluted with chloroform, washed with water, and the solvent removed under reduced pressure. The residue was chromatographed on a silica flash column eluting with dichloromethane. A green fraction was collected and recrystallized from chloroform-hexanes to give the silver(III) complex (3.8 mg, $43 \%)$ as dark crystals, mp $>300{ }^{\circ} \mathrm{C}$; UV-Vis $\left(\mathrm{CHCl}_{3}\right): \lambda_{\max }\left(\log _{10} \varepsilon\right) 390$ (4.89), 445 (4.88), 506 (4.29), 543 (4.28), $599 \mathrm{~nm}(4.19) ;{ }^{1} \mathrm{H} \mathrm{NMR}\left(\mathrm{CDCl}_{3}\right): \delta 1.55(6 \mathrm{H}, \mathrm{t}, J=7.6 \mathrm{~Hz}), 3.18(6 \mathrm{H}, \mathrm{s})$, $3.56(4 \mathrm{H}, \mathrm{q}, J=7.6 \mathrm{~Hz}), 6.93-6.96(2 \mathrm{H}, \mathrm{m}), 7.17-7.20(2 \mathrm{H}, \mathrm{m}), 7.51-7.86(10 \mathrm{H}, \mathrm{m}), 8.01(2 \mathrm{H}$, s), $9.23(2 \mathrm{H}, \mathrm{s}) ;{ }^{13} \mathrm{C} \mathrm{NMR}\left(\mathrm{CDCl}_{3}\right): \delta 11.5,16.8,20.0,29.9,98.8,100.5,121.8,127.3,128.6$, 132.2, 135.5, 135.6, 136.1, 136.6, 140.7, 141.4, 141.8, 143.5, 145.3, 145.4; HRMS (EI): calcd for $\mathrm{C}_{41} \mathrm{H}_{34} \mathrm{~N}_{3} \mathrm{Ag}$ : 675.1803; found: 675.1793 . 